\title{
Special issue with selected papers from PODC 2012
}

\author{
Alessandro Panconesi
}

Published online: 8 January 2015

(C) Springer-Verlag Berlin Heidelberg 2015

This special issue of Distributed Computing contains four papers that were considered to be particularly praiseworthy by the Program Committee of PODC 2012, the 31st Annual ACM SIGACT-SIGOPS Symposium on Principles of Distributed Computing. PODC 2012 took place in the island of Madeira, a very beautiful setting but not the easiest one to organize an international conference. Luis Rodrigues and Oksana Denysyuk went above and beyond the call of duty to ensure that everything went smoothly, and it did. I would like to thank them on behalf of all participants.

The conference itself was intellectually very stimulating, even by the high standards of PODC. The papers in this special issue are a good sample of the variety of topics that were discussed. The paper "Wait-freedom with advice" provides new insights into the fascinating world of failure detectors. It introduces a new characterization of detectors that is recommended reading for anybody interested in this elegant and useful theory. "Faster randomized consensus with an oblivious adversary" shows new strong upper bounds for this fundamental problem when the environment is modelled as an oblivious adversary, a scenario whose computational complexity was previously not well understood. "Weak Models of Distributed Computing, with Connections to Modal Logic" shows an interesting connection between lower-bounds for distributed graph algorithms and modal logic, whose machinery is used to derive several impossibility results. The understanding of information diffusion over dynamic graphs, i.e. graphs that change their topology over time, is a fascinating topic that acquires urgency in a world increasingly permeated by social media. "Information Spreading in Dynamic Graphs" presents an elegant and general bound for the flooding time in terms of the mixing time of the Markov process that governs the evolution of the graph. All papers, I am sure, make an enjoyable and thought provoking reading.

Two additional papers were invited to the special issue but, due to time constraints, they could not appear in this volume. If accepted, they will appear in a future volume of this journal.

I would like to thank the colleagues of the PODC 2012 program committee for their outstanding contribution, and Hagit Attiya, editor-in-chief of Distributed Computing, for her energetic and efficient help with the preparation of this special issue.

Alessandro Panconesi, PODC 2012 Program Committee Chair

\footnotetext{
A. Panconesi $(\varangle)$

Computer Science Department, Sapienza University of Rome,

via Salaria 113, 00198 Roma, Italy

e-mail: ale@di.uniroma1.it
} 